\title{
Consensus control of multi-agent systems with input delays: a descriptor model approach
}

\author{
Lyubchyk L. M., Dorofieiev Y. I. \\ National Technical University "Kharkiv Polytechnic Institute", \\ 2 Kirpicheva Str., 61002, Kharkiv, Ukraine
}

(Received 15 October 2019; Revised 13 November 2019; Accepted 15 November 2019)

\begin{abstract}
This paper addresses the consensus control design for multi-agent systems with input time delay, which is unknown but bounded. Descriptor transformation is used to obtain a model without delay for closed-loop individual agent. The sufficient conditions for global consensus under directed communication topology are identified using Lyapunov-Krasovskii functional. To analyze the stability of the networked multi-agent system based on the relationship between the agents, the Lyapunov function method using corresponding comparison system is used. The effectiveness of the proposed control design method is demonstrated by a numerical simulation example.
\end{abstract}

Keywords: multi-agent system, consensus control, descriptor model, Lyapunov-Krasovskii functional, linear matrix inequality, semidefinite programming.

2000 MSC: 34K35, 90C22, 93D30

UDC: 519.71

DOI: $10.23939 / \mathrm{mmc} 2019.02 .333$

\section{Introduction}

A multi-agent system (MAS) is used as a model of a large number of controlled complex systems with network structures, such as robotics and manufacturing systems, logistics systems and supply chains, distributed computing systems, and many others.

Among the networked MAS control problems, the problem of consensus control is of particular interest and attracts the attention of a large number of researchers. The main aspect of the consensus problem is to make the MAS network reach an agreement of pre-specified variables in a cooperative way using local information in the form of distributed protocol. Numerous results have been reported in the literature for the design of distributed consensus protocols for MAS, see $[1,2]$ and references therein.

The structure of MAS is described by communication topology model in the form of directed graph with nodes described controlled individual agents, and the edges represent transfer of information between them. Control of MAS is carried out on the basis of a consensus protocol [3], which refers to the formation of local agent control based on the comparison of the information on the state of the agent and its neighbor states, in accordance with communication topology. The consensus problem in MAS is to align states of individual agents to each other by constructing such a consensus protocol which ensures bringing the states of all agents to the same constant value.

Generally, the consensus protocol is chosen as linear feedbacks deviation states or outputs of the corresponding agent from the weighted average of the state vectors or outputs of its immediate neighbors. In this case, the problem reduces to finding a set of feedback gain matrices from the condition of stability of both individual controlled agents and the system as a whole.

The usual approach to solving such a problem is to construct an extended dynamic model of MAS with respect to the composite systems state vector composed of state vectors of individual agents; thus to construct a model uses the concept of the Laplacian of communication topology graph. At that, the MAS dynamic matrix is described by the Kronecker product of the corresponding matrices [4]. In 
this approach, a consensus control synthesis based on the conditions of stability of a high-dimensional matrix of special structure and associated with certain computational difficulties.

Time-delay is an important feature that should be taken into account when solving the MAS control problem, it is almost inevitable and its presence can lead to undesirable performance and even to instability. Its relevance does not decrease for MAS, and as first shown by Olfati-Saber and Murray [5] it can prevent the network to reach consensus. In this paper, authors had started the analysis of conditions of consensus attainment in MAS with constant uniform time-delays and provided a necessary and sufficient condition for the upper bound of time delays under the assumption that all the delays are equal and time-invariant, later their research was expanded for different types of delays. Sufficient conditions were given in [6] for average consensus with constant, time-varying and nonuniform time delays. In [7], the output consensus for MAS with different types of time delays including communication delay, identical self-delay, and different self-delay was studied. In [8], discretetime multi-agent systems with dynamically changing topologies and time-varying communication delays were considered. In [9], the Artstein reduction method was applied to deal with continuous-time MAS with delay, which is constant but unknown, and further endeavors are made to ensure the extra integral term of the system state due to the uncertainties using Lyapunov-Krasovskii functionals. By transforming the Laplacian matrix into the real Jordan form, the global stability analysis was put in the framework of the Lyapunov stability theory.

In this paper, we consider a decentralized approach to consensus control synthesis, including local stabilizing controllers design for each agent of the networked system and synthesis of consensus controllers to ensure the conditions of agent's coordination. Synthesis of local regulators for individual agents is based on the method of invariant ellipsoids [10] and reduced to solving a system of linear matrix inequalities. To analyze the stability of the MAS taking into account the relationship between the agents, the method of vector Lyapunov functions is used [11,12], which leads to the problem of reference system stability analysis, the dimension of which coincides with the number of agents. At the same time, the synthesis of consensus controllers is performed using the obtained stability conditions for the reference system for given consensus protocol. Thus, the decomposition approach to consensus control problem in MAS, in contrary to traditional solutions, leads to the stabilizing and consensus controllers design problem of the reduced dimension, which is essential for complex network structure systems.

\section{Problem statement}

Consider MAS as a network of $N$ agents with linear or linearized dynamics in the discrete-time setting, where the dynamics of the $i$-th agent are described by discrete state equations

$$
x_{i}(k+1)=\mathrm{A} x_{i}(k)+\mathrm{B} u_{i}\left(k-\lambda_{i}(k)\right), \quad i=\overline{1, N},
$$

where $k=0,1, \ldots$ is time instant; $x_{i}(k) \in \mathbb{R}^{n}$ is the state of $i$-th agent at the time $k ; u_{i}(k) \in \mathbb{R}^{m}$ is control input; $\mathrm{A} \in \mathbb{R}^{n \times n}$ and $\mathrm{B} \in \mathbb{R}^{n \times m}$ are constant matrices with (A, B) being controllable; $\lambda_{i}(k)>0$ is the input delay, which is assumed unknown but satisfies the inequality $0 \leqslant \lambda_{i}(k) \leqslant \lambda_{i}^{\max }$, where $\lambda_{i}^{\max }$ is known. The initial values are given by $x_{i}(0), u_{i}(-1), \ldots, u_{i}\left(-\lambda_{i}^{\max }\right)$.

The communication topology of MAS network is represented by directed graph $G=(V, E)$, where $V=\{1, \ldots, N\}$ is the set of nodes (i.e., agents) and $E \subset V \times V$ is the set of edges, reflecting information transfer between agents. An edge $(i, j)$ in graph $G$ means that agents $i$ and $j$ exchange information.

As far as information is exchanged between every two agents which are connected by one edge through the communication channel, it is necessary to take time delays of message transmission into account. The $i$-th agent can use its own state information directly without delay, and its neighbour's lagged states

$$
u_{i}(k)=\Sigma_{i} \mathrm{~K}_{i} \sum_{\substack{j=1 \\ j \neq i}}^{N} d_{i j}\left(x_{i}(k)-x_{j}\left(k-\lambda_{i j}(k)\right)\right), \quad \Sigma_{i}=\frac{1}{\sum_{\substack{j=1 \\ j \neq i}}^{N} d_{i j}},
$$


where $\mathrm{D}=\left\{d_{i j}\right\} \in \mathbb{R}^{N \times N}$ is the adjacency matrix associated with graph $G ; \lambda_{i j}(k)>0$ is information delay between nodes $i$ and $j ; \mathrm{K}_{i} \in \mathbb{R}^{m \times n}$ is the state feedback control gain matrix to be determined, this kind of protocols have received many attention in studies [13-15].

Consider consensus problems for second-order linear systems, where the time delay affects only the state information that is being transmitted. However, the consensus for protocol (2) for a general system with nonuniform time delays remains unknown.

For MAS (1) the protocol (2) is said to solve the consensus problem if

$$
\left\|x_{i}(k)-x_{j}(k)\right\| \rightarrow 0 \quad \text { as } \quad k \rightarrow \infty \quad \forall i, j=\overline{1, N},
$$

that reflects the conditions of coordinated behavior of individual agents.

Thus, the consensus problem of control is to select matrix $\mathrm{K}_{i}$, which ensure stabilization of the closed-loop subsystem, stability of controlled MAS altogether and the fulfillment of consensus conditions (3). In addition, it puts forward the requirement of control synthesis procedures decomposition providing for independent determination of these matrices based on the aggregation of local agents control subsystems.

\section{Descriptor model of networked MAS}

Substituting protocol equations (2) into system (1) yields the following system

$$
x_{i}(k+1)=\left(\mathrm{A}+\mathrm{BK}_{i}\right) x_{i}(k)-\Sigma_{i} \mathrm{BK}_{i} \sum_{\substack{j=1 \\ j \neq i}}^{N} d_{i j} x_{j}\left(k-\lambda_{i j}(k)\right) .
$$

The local performance criterion in the case of an infinite time horizon is chosen in the form

$$
J_{i}(k)=\sum_{t=k}^{\infty} \beta^{t}\left(x_{i}^{\mathrm{T}}(t) \mathrm{W}_{i}^{x} x_{i}(t)+u_{i}^{\mathrm{T}}(t) \mathrm{W}_{i}^{u} u_{i}(t)\right),
$$

where $\mathrm{W}_{i}^{x} \in \mathbb{R}^{n \times n}$ and $\mathrm{W}_{i}^{u} \in \mathbb{R}^{m \times m}$ are positive definite diagonal weight matrices; $0<\beta<1$ is discount coefficient.

If during the construction of control objects models additional state variables are introduced that are algebraically related to the main variables, the systems are called descriptor models [16]. Due to the presence of additional algebraic relations between state variables, descriptor models acquire properties that are not characteristic of the traditional way of describing systems, which provides the developer with additional capabilities.

In order to obtain a model without delay, perform the descriptor transformation [17] of the model (4). Introduce an additional variable $y_{i}(k)=x_{i}(k+1)-x_{i}(k)$. It is easy to verify that equality holds

$$
x_{i}\left(k-\lambda_{i j}(k)\right)=x_{i}(k)-\sum_{l=k-\lambda_{i j}^{\max }}^{k-1} y_{j}(l) .
$$

The closed-loop model (4) taking into account (6), is represented in the form

$$
\left[\begin{array}{c}
x_{i}(k+1) \\
0_{n \times 1}
\end{array}\right]=\left[-y_{i}(k)+\left(\mathrm{A}-\mathrm{I}_{n}+\mathrm{B} \mathrm{K}_{i}\right) x_{i}(k)+\Sigma_{i} \mathrm{~B} \mathrm{~K}_{i} \sum_{\substack{j=1 \\
j \neq i}}^{N} d_{i j}\left(-x_{j}(k)+\sum_{l=k-\lambda_{i j}^{\max }}^{k-1} y_{j}(l)\right)\right],
$$

where $0_{n \times m}$ and $\mathrm{I}_{n}$ are zero and identity matrices of the corresponding dimension.

Mathematical Modeling and Computing, Vol. 6, No. 2, pp. 333-343 (2019) 
Introduce a composite state vector $\xi_{i}(k)=\operatorname{col}\left\{x_{1}(k), \ldots, x_{N}(k), y_{i}(k)\right\}$ of the descriptor model $(7)$ with notation:

$$
\begin{aligned}
& \mathrm{E}=\left[\begin{array}{cccc}
\mathrm{I}_{n} & 0_{n \times n} & \cdots & 0_{n \times n} \\
0_{n \times n} & 0_{n \times n} & \cdots & 0_{n \times n}
\end{array}\right] \in \mathbb{R}^{2 n \times(N+1) n}, \\
& \overline{\mathrm{A}}_{i}=\left[\begin{array}{ccccc}
\mathrm{I}_{n} & 0_{n \times n} & \cdots & 0_{n \times n} & \mathrm{I}_{n} \\
\mathrm{~A}-\mathrm{I}_{n}+\mathrm{BK}_{i} & -d_{i 2} \Sigma_{i} \mathrm{BK}_{i} & \cdots & -d_{i N} \Sigma_{i} \mathrm{~B} \mathrm{~K}_{i} & -\mathrm{I}_{n}
\end{array}\right] \in \mathbb{R}^{2 n \times(N+1) n}, \\
& \overline{\mathrm{B}}_{i}=\left[\begin{array}{c}
0_{n \times n} \\
\Sigma_{i} \mathrm{~B} \mathrm{~K}_{i}
\end{array}\right] \in \mathbb{R}^{2 n \times n} .
\end{aligned}
$$

Finally, get the descriptor model of the node with control (2) in the form

$$
\mathrm{E} \xi_{i}(k+1)=\overline{\mathrm{A}}_{i} \xi_{i}(k)+\overline{\mathrm{B}}_{i} \sum_{\substack{j=1 \\ j \neq i}}^{N} d_{i j} \sum_{l=k-\lambda_{i j}^{\max }}^{k-1} y_{j}(l) .
$$

Thus, if the sequence $x_{i}(k), k=0,1, \ldots$ is a solution to system (1), then the sequence $\xi_{i}(k), k=0,1, \ldots$ is a solution to system (8) and vice versa.

\section{Synthesis of consensus control}

In order the gain matrices choosing we use an approach that is based on the sufficient conditions for stability using the Lyapunov-Krasovskii functional (LKF) for each $i$-th agent. Define the block matrix

$$
\mathrm{P}_{i}=\left[\begin{array}{ccccc}
\mathrm{P}_{1} & 0 & 0 & \cdots & 0 \\
\mathrm{P}_{2} & \mathrm{P}_{3} & 0 & \cdots & 0 \\
0 & 0 & \mathrm{I} & \cdots & 0 \\
\vdots & \vdots & \vdots & \ddots & \vdots \\
0 & 0 & 0 & \cdots & \mathrm{I}
\end{array}\right]
$$

where $0 \prec \mathrm{P}_{1}=\mathrm{P}_{1}^{\mathrm{T}} \in \mathbb{R}^{n \times n}, \mathrm{P}_{2}, \mathrm{P}_{3} \in \mathbb{R}^{n \times n}$, and construct the LKF for the descriptor system (8) in the form

$$
\begin{gathered}
V_{i}(k)=V_{i}^{x}(k)+\sum_{\substack{j=1 \\
j \neq i}}^{N} V_{i j}^{y}(k), \quad V_{i}^{x}(k)=\beta^{k} \xi_{i}^{\mathrm{T}}(k) \mathrm{EP}_{i} \mathrm{E}_{i}(k)=\beta^{k} x_{i}^{\mathrm{T}}(k) \mathrm{P}_{1} x_{i}(k), \\
V_{i j}^{y}(k)=\beta^{k} \sum_{r=-\lambda_{i j}^{\text {max }}}^{-1} \sum_{t=k+r}^{k-1} y_{j}^{\mathrm{T}}(t) \mathrm{Z}_{j} y_{j}(t), \quad \mathrm{Z}_{j}=\mathrm{Z}_{j}^{\mathrm{T}} \succ 0, \quad j=\overline{1, N}, \quad j \neq i .
\end{gathered}
$$

Calculate the first difference by $k$ LKF (9)-(10) due to the system (8)

$$
\begin{aligned}
\Delta V_{i}^{x}(k)= & V_{i}^{x}(k+1)-V_{i}^{x}(k)=\beta^{k}\left[\beta x_{i}^{\mathrm{T}}(k)\left(\mathrm{A}+\mathrm{B} \mathrm{K}_{i}\right)^{\mathrm{T}} \mathrm{P}_{1}\left(\mathrm{~A}+\mathrm{B} \mathrm{K}_{i}\right) x_{i}(k)\right. \\
& -\beta x_{i}^{\mathrm{T}}(k)\left(\mathrm{A}+\mathrm{B} \mathrm{K}_{i}\right)^{\mathrm{T}} \mathrm{P}_{1} \Sigma_{i} \mathrm{~B} \mathrm{~K}_{i} \sum_{\substack{j=1 \\
j \neq i}}^{N} d_{i j} x_{j}(k)-\beta \sum_{\substack{j=1 \\
j \neq i}}^{N} d_{i j} x_{j}^{\mathrm{T}}(k) \Sigma_{i}\left(\mathrm{~B} \mathrm{~K}_{i}\right)^{\mathrm{T}} \mathrm{P}_{1}\left(\mathrm{~A}+\mathrm{B} \mathrm{K}_{i}\right) x_{i}(k) \\
& \left.+\beta \sum_{\substack{j=1 \\
j \neq i}}^{N} d_{i j} x_{j}^{\mathrm{T}}(k) \Sigma_{i}\left(\mathrm{~B} \mathrm{~K}_{i}\right)^{\mathrm{T}} \mathrm{P}_{1} \Sigma_{i} \mathrm{~B} \mathrm{~K}_{i} \sum_{\substack{j=1 \\
j \neq i}}^{N} d_{i j} x_{j}(k)-x_{i}^{\mathrm{T}}(k) \mathrm{P}_{1} x_{i}(k)\right]
\end{aligned}
$$

Mathematical Modeling and Computing, Vol. 6, No. 2, pp. 333-343 (2019) 


$$
\begin{aligned}
\Delta V_{i j}^{y}(k) & =V_{i j}^{y}(k+1)-V_{i j}^{y}(k)=\beta^{k}\left[\lambda_{i j}^{\max } y_{j}^{\mathrm{T}}(k) \mathrm{Z}_{j} y_{j}(k)-\sum_{t=k-\lambda_{i j}^{\max }}^{k-1} y_{j}^{\mathrm{T}}(t) \mathrm{Z}_{j} y_{j}(t)\right] \\
& =\beta^{k}\left[\lambda_{i j}^{\max } y_{j}^{\mathrm{T}}(k) \mathrm{Z}_{j} y_{j}(k)-x_{j}^{\mathrm{T}}(k) \mathrm{Z}_{j} x_{j}(k)+x_{j}^{\mathrm{T}}\left(k-\lambda_{i j}^{\max }\right) \mathrm{Z}_{j} x_{j}\left(k-\lambda_{i j}^{\max }\right)\right] .
\end{aligned}
$$

As a result, in the case $i=1$ was received

$$
\Delta V_{1}(k)=\Delta V_{1}^{x}(k)+\sum_{j=2}^{N} \Delta V_{1 j}^{y}(k)=\beta^{k} s_{1}^{\mathrm{T}}(k) \Gamma\left(\lambda_{1 j}^{\max }\right) s_{1}(k),
$$

where $s_{1}(k)=\operatorname{col}\left\{x_{1}(k), \mathrm{BK}_{1} x_{2}(k), \ldots, \mathrm{BK}_{1} x_{N}(k), x_{2}\left(k-\lambda_{12}^{\max }\right), \ldots, x_{N}\left(k-\lambda_{1 N}^{\max }\right), y_{2}(k), \ldots, y_{N}(k)\right\}$;

$$
\Gamma\left(\lambda_{1 j}^{\max }\right)=\left[\begin{array}{cccccc}
\beta \Psi_{1}^{\mathrm{T}} \mathrm{P}_{1} \Psi_{1}-\mathrm{P}_{1} & -\beta d_{12} \Sigma_{1} \Psi_{1}^{\mathrm{T}} \mathrm{P}_{1} & \cdots & -\beta d_{1 N} \Sigma_{1} \Psi_{1}^{\mathrm{T}} \mathrm{P}_{1} & 0_{n \times(N-1) n} & 0_{n \times(N-1) n} \\
* & \beta d_{12}^{2} \Sigma_{1}^{2} \mathrm{P}_{1}-\mathrm{Z}_{2} & \cdots & -\beta d_{12} d_{1 N} \Sigma_{1}^{2} \mathrm{P}_{1} & 0_{n \times(N-1) n} & 0_{n \times(N-1) n} \\
* & * & \ddots & \vdots & 0_{n \times(N-1) n} & 0_{n \times(N-1) n} \\
* & * & * & \beta d_{1 N}^{2} \Sigma_{1}^{2} \mathrm{P}_{1}-\mathrm{Z}_{N} & 0_{n \times(N-1) n} & 0_{n \times(N-1) n} \\
* & * & * & * & \mathbb{Z} & 0_{(N-1) n \times(N-1) n} \\
* & * & * & * & * & \mathbb{Z}_{\lambda}
\end{array}\right],
$$

the symbol "*" denotes the corresponding block in the symmetric matrix of the inequality; $\Psi_{1}=$ $\mathrm{A}+\mathrm{B} \mathrm{K}_{1} ; \mathbb{Z}=$ block $\operatorname{diag}\left\{\mathrm{Z}_{2}, \ldots, \mathrm{Z}_{N}\right\} ; \mathbb{Z}_{\lambda}=\operatorname{block} \operatorname{diag}\left\{\lambda_{12}^{\max } \mathrm{Z}_{2}, \ldots, \lambda_{1 N}^{\max } \mathrm{Z}_{N}\right\}$.

The stability condition is a decrease of the LKF (9)-(10) value on the trajectories of system (8) over time with a certain guaranteed rate determined by the current value of criterion (5)

$$
\Delta V_{i}(k) \leqslant-\beta^{k} x_{i}^{\mathrm{T}}(k)\left(\mathrm{W}_{i}^{x}+\mathrm{K}_{i}^{\mathrm{T}} \mathrm{W}_{i}^{u} \mathrm{~K}_{i}\right) x_{i}(k) .
$$

Summing the left and right sides of inequality (11) from 0 to $\infty$, we obtain

$$
J_{i}(k) \leqslant x_{i}^{\mathrm{T}}(0) \mathrm{P}_{1} x_{i}(0)+\sum_{\substack{j=1 \\ j \neq i}}^{N}\left(\lambda_{i j}^{\max } y_{j}^{\mathrm{T}}(0) \mathrm{Z}_{j} y_{j}(0)-\sum_{t=-\lambda_{i j}^{\max }}^{-1} y_{j}^{\mathrm{T}}(t) \mathrm{Z}_{j} y_{j}(t)\right),
$$

that is, LKF (9)-(10), calculated at the time instant $k=0$, determines the upper boundary value of criterion (5). Then the consensus control synthesis problem is equivalent to

$$
u_{i}(k)=\arg \min _{u_{i}(k)} V(0) .
$$

The classical approach to solving the control synthesis problem that minimizes the quadratic quality criterion is based on the solution of the Riccati algebraic equation and guarantees an optimal solution for arbitrary initial conditions. By this, the control obtained as a result of solving problem (13) differs from the classical one. Modify proposed approach to the purpose of initial conditions elimination, using invariant ellipsoid method [10].

The ellipsoid described by the equation

$$
\varepsilon_{i}\left(\mathrm{Q}_{i}\right)=\left\{x \in \mathbb{R}^{n}: x_{i}^{\mathrm{T}}(k) \mathrm{Q}_{i}^{-1} x_{i}(k) \leqslant 1\right\},
$$

where $\mathrm{Q}_{i} \in \mathbb{R}^{n \times n}$ is matrix of the ellipsoid, is called state invariant for the considered system if any trajectory of the system, starting in an ellipsoid, remains in it for any time instant $k \geqslant 0$.

By analogy with (14), define a family of ellipsoids that are invariant by state with delay

$$
\varepsilon_{j t}\left(\mathrm{R}_{j}\right)=\left\{x \in \mathbb{R}^{n}: x_{j}^{\mathrm{T}}(k-t) \mathrm{R}_{j}^{-1} x_{j}(k-t) \leqslant 1\right\}, \quad t=\overline{1, \lambda_{i j}^{\max }} .
$$


The sum and difference of ellipsoids is considered in the sense of the sum and difference of sets according to Minkowski [18]. Then the sum of the invariant ellipsoid (14) and the family of ellipsoids (15) can be considered as an approximation of the reachability set of the descriptor system (8), i.e., it allows characterizing the influence of external perturbations and time delays on the system trajectory. The second term in the LKF (9)-(10) can be represented as

$$
V_{i j}^{y}(k)=\beta^{k}\left[\lambda_{i j}^{\max } x_{j}^{\mathrm{T}}(k) \mathrm{Z}_{j} x_{j}(k)-\sum_{t=-\lambda_{i j}^{\max }}^{-1} x_{j}^{\mathrm{T}}(k-t) \mathrm{Z}_{j} x_{j}(k-t)\right] .
$$

A comparison of expressions (9) and (14), as well as (16) and (15), allows us to state that if the conditions are satisfied

$$
\mathrm{P}_{1}=\mathrm{Q}_{i}^{-1}, \quad \mathrm{Z}_{j}=\mathrm{R}_{j}^{-1}, \quad j=\overline{1, N}, \quad j \neq i
$$

then the sum of the ellipsoid (14) and the family of ellipsoids (15) represents a set that can be considered as an upper estimate of the level set of the LKF (9)-(10).

Thus, the problem is reduced to constructing a controller that ensures minimization, according to some criterion, of the sum of invariant ellipsoids. As a criterion, the sum of the squares of the ellipsoids semiaxes is selected, that is, the sum of the trace of the matrix $Q_{i}$ and the traces of the matrices $\mathrm{R}_{j}$.

The result of solving the consensus control synthesis problem for MAS with unknown but bounded delays is presented in the form of a theorem.

Theorem 1. For descriptor system (8), if the matrices $\hat{\mathrm{Q}}_{i} \in \mathbb{R}^{n \times n}, \hat{\mathrm{Y}}_{i} \in \mathbb{R}^{m \times n}$ and $\hat{\mathrm{R}}_{j} \in \mathbb{R}^{n \times n}$, $j=\overline{1, N}$ are obtained as a result of solving the problem

$$
\operatorname{tr} \mathrm{Q}_{i}+\sum_{j=1, j \neq 1}^{N} \operatorname{tr} \mathrm{R}_{j} \rightarrow \min
$$

under constraint on matrix variables $0 \prec \mathrm{Q}_{i}=\mathrm{Q}_{i}^{\mathrm{T}} \in \mathbb{R}^{n \times n}, \mathrm{Y}_{i} \in \mathbb{R}^{m \times n}$ and $0 \prec \mathrm{R}_{j}=\mathrm{R}_{j}^{\mathrm{T}} \in \mathbb{R}^{n \times n}$

$$
\left[\begin{array}{ccccccc}
-\mathrm{Q}_{1} & \beta^{\frac{1}{2}}\left(\mathrm{AQ}_{1}+\mathrm{BY}_{1}\right) & \Phi_{1} & 0_{n \times(N-1) n} & 0_{n \times(N-1) n} & 0_{n \times n} & 0_{n \times m} \\
* & -\mathrm{Q}_{1} & 0_{n \times(N-1) n} & 0_{n \times(N-1) n} & 0_{n \times(N-1) n} & \mathrm{Q}_{1} \mathrm{~W}_{1}^{x \frac{1}{2}} & \mathrm{Y}_{1}^{\mathrm{T}} \mathrm{W}_{1}^{u \frac{1}{2}} \\
* & * & -\mathbb{R} & 0_{(N-1) n \times(N-1) n} & 0_{(N-1) n \times(N-1) n} & 0_{(N-1) n \times n} & 0_{(N-1) n \times m} \\
* & * & * & \mathbb{R} & 0_{(N-1) n \times(N-1) n} & 0_{(N-1) n \times n} & 0_{(N-1) n \times m} \\
* & * & * & * & \mathbb{R}_{\lambda} & 0_{(N-1) n \times n} & 0_{(N-1) n \times m} \\
* & * & * & * & * & -\mathrm{I}_{n} & 0_{n \times m} \\
* & * & * & * & * & * & -\mathrm{I}_{m}
\end{array}\right]
$$

where

$$
\begin{gathered}
\Phi_{1}=\left[\left(\beta d_{12} \Sigma_{1}\right)^{\frac{1}{2}} \mathrm{R}_{2}|\cdots|\left(\beta d_{1 N} \Sigma_{1}\right)^{\frac{1}{2}} \mathrm{R}_{N}\right], \quad \mathbb{R}_{\lambda}=\text { block diag }\left\{\lambda_{12}^{\max } \mathrm{R}_{2}, \ldots, \lambda_{1 N}^{\max } \mathrm{R}_{N}\right\} \\
\mathbb{R}=\text { block } \operatorname{diag}\left\{\mathrm{R}_{2}, \ldots, \mathrm{R}_{N}\right\}
\end{gathered}
$$

then:

i) for any initial state values the closed system (8) is asymptotically stable;

ii) among all consensus protocol of the form (2), a protocol with a control gain matrix

$$
\mathrm{K}_{i}=\hat{\mathrm{Y}}_{i} \hat{\mathrm{Q}}_{i}^{-1}
$$

affords a minimum to the sum of the invariant ellipsoid (14) and the family of ellipsoids (15) by the criterion of the matrix trace. 
Proof. Let represent inequality (11), which ensures the decrease of the LKF (9)-(10) along any trajectory of system (8), in the form of a linear matrix inequality (LMI), which can be written as the sum

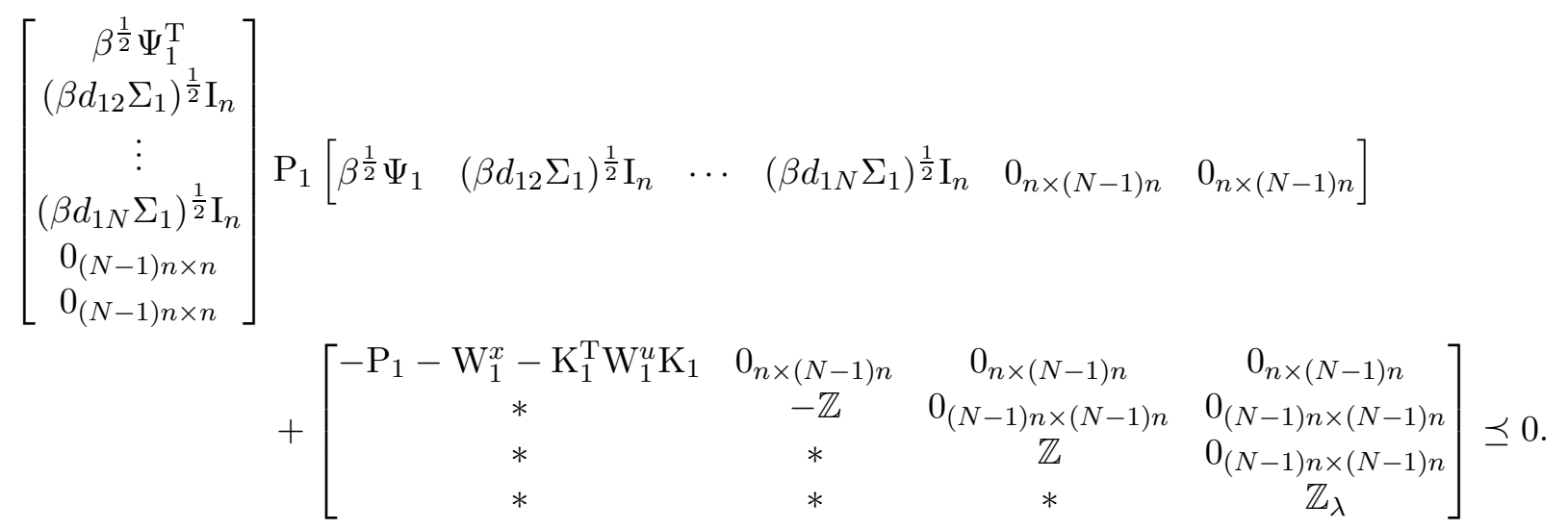

Consider the matrix variables $\mathrm{Y}_{i}=\mathrm{K}_{i} \mathrm{Q}_{i}$, then, by virtue $\mathrm{Q}_{i} \succ 0$, the matrix $\mathrm{K}_{i}$ is restored monosemantic as $\mathrm{K}_{i}=\mathrm{Y}_{i} \mathrm{Q}_{i}^{-1}$. Using the Schur complement lemma [19] and applying congruent transformation to the obtained inequality matrix using the block-diagonal matrix block $\operatorname{diag}\left\{\mathrm{I}_{n}, \mathrm{P}_{1}^{-1}, \mathrm{Z}_{2}^{-1}, \ldots\right.$, $\left.\mathrm{Z}_{N}^{-1}, \mathrm{Z}_{2}^{-1}, \ldots, \mathrm{Z}_{N}^{-1}, \mathrm{Z}_{2}^{-1}, \ldots, \mathrm{Z}_{N}^{-1}\right\}$ we represent the inequality as LMI (19) taking into account (17). The upper boundary value of the quality criterion in (12) depends on the initial conditions of system (8). To eliminate this dependence, we apply the approach proposed in [20]. Suppose that the initial state of each agent is arbitrary, but belongs to an ellipsoid

$$
\varepsilon_{i}\left(\mathrm{Q}_{i}\right)=\left\{x \in \mathbb{R}^{n}: x_{i}^{\mathrm{T}}(-k) \mathrm{Q}_{i}^{-1} x_{i}(-k) \leqslant 1, k=0,1, \ldots, \lambda_{i}^{\max }\right\},
$$

whose matrix is calculated as a result of solving the problem (18) under constraint (19). Thus, if the indicated problem has an acceptable solution $\hat{\mathrm{Q}}_{i}, \hat{\mathrm{Y}}_{i}$ and $\hat{\mathrm{R}}_{j}, j=\overline{1, N}$, then descriptor system (8) is asymptotically stable.

Remark 1. Since the descriptor system (8) is equivalent to the closed-loop system (4), the solvability of problem (18) also guarantees the asymptotic stability of input-delayed multi-agent system (1) as well as the control law in the form

$$
u_{i}(k)=\Sigma_{i} \hat{\mathrm{Y}}_{i} \hat{\mathrm{Q}}_{i}^{-1} \sum_{\substack{j=1 \\ j \neq i}}^{N} d_{i j}\left(x_{i}(k)-x_{j}\left(k-\lambda_{i j}(k)\right)\right)
$$

ensures that the consensus condition (3) is satisfied.

Remark 2. The optimization problem (18) under constraint (19) is a semidefinite problem and is solved numerically using freely distributed software packages developed on the basis of the MATLAB environment, for example, the cvx [21].

\section{Stability analysis of networked MAS}

To analyze the stability of the networked MAS based on the relationship between the agents the method of vector Lyapunov functions [11] using corresponding comparison system [12] is used as it was proposed in [22]. Introduce composite vector $x(k)=\operatorname{col}\left\{x_{1}(k), \ldots, x_{N}(k)\right\}$ as a state vector of considered MAS and construct vector Lyapunov function (VLF)

$$
V_{\text {vect }}(k)=\operatorname{col}\left\{V_{1}(k), \ldots, V_{N}(k)\right\},
$$

components of which are LKF of local agents in the form (9)-(10). Using VLF (22) a general Lyapunov function for overall MAS is constructed 


$$
V(k)=\sum_{i=1}^{N} p_{i} V_{i}(k)=P V_{\text {vect }}(k), \quad P=\operatorname{raw}\left\{p_{1}, \ldots, p_{N}\right\}, \quad p_{i}>0 .
$$

In accordance with [12], the linear aggregated comparison system, associates with a set of agents of MAS, defined by difference equations

$$
\begin{aligned}
& \nu(k+1)=\Lambda \nu(k), \quad \nu(0)=V(0), \\
& \eta(k)=P \nu(k),
\end{aligned}
$$

where $\nu(k) \in \mathbb{R}^{N}$ is comparison system state vector; $\nu(k)$ is scalar function, which is the output of the comparison system; $\Lambda \in \mathbb{R}^{N \times N}$ is comparison system dynamics matrix.

Matrix $\Lambda$ elements may be treated as follows: the diagonal elements $[\Lambda]_{i i}, i=\overline{1, N}$ give an upper bound of $i$-th agents transmission coefficients, and each elements $[\Lambda]_{i j}, i, j=\overline{1, N}, j \neq i$ give an upper estimate of the coupling coefficient between $i$-th and $j$-th agents. To calculate the matrix $\Lambda$ elements, the approach proposed in the work [22] is used.

Vector (22) and general (23) Lyapunov functions of the MAS under consideration satisfy the inequalities

$$
V_{\text {vect }}(k) \leqslant \nu(k), \quad V(k) \leqslant \eta(k) .
$$

Thus, comparison system (24) components dominates vector (22) and general (23) Lyapunov functions. As a result, the stability of linear aggregated comparison system (24) guarantees the stability of considered MAS. Therefore, if the stability condition

$$
\rho(\Lambda)<1
$$

where $\rho(\Lambda)$ is spectral radius of comparison system (24) dynamics matrix, is satisfied, the networked MAS is Schur stable and, consequently, the consensus control (21) ensures the fulfillment of the target condition (3).

\section{Numerical example}

As an example, consider discrete MAS of 6 agents, which was studied in [23] and the solution of consensus control problem, using proposed descriptor model approach. The communication graph describing networked MAS structure is shown in Fig. 1.

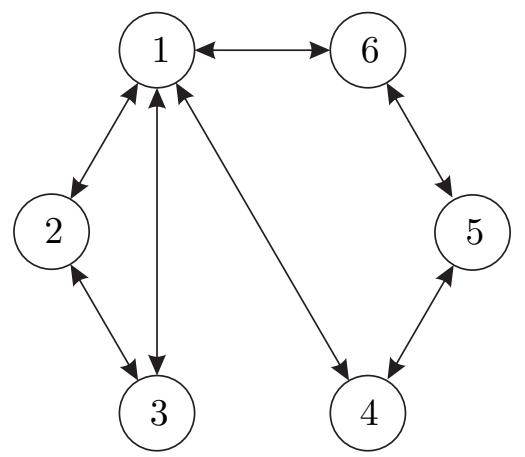

Fig. 1. Structure of the MAS.

The adjacency matrix of MAS communication topology graph is

$$
\mathrm{D}=\left[\begin{array}{llllll}
0 & 1 & 1 & 1 & 0 & 1 \\
1 & 0 & 1 & 0 & 0 & 0 \\
1 & 1 & 0 & 0 & 0 & 0 \\
1 & 0 & 0 & 0 & 1 & 0 \\
0 & 0 & 0 & 1 & 0 & 1 \\
1 & 0 & 0 & 0 & 1 & 0
\end{array}\right] .
$$

Dimensions of the model are: $n=2$ and $m=1$. The agent dynamics and the consensus protocol are given by (1) and (2), respectively, with $\mathrm{A}=\left[\begin{array}{cc}0 & 1 \\ -1 & 1.02\end{array}\right], \mathrm{B}=\left[\begin{array}{l}1 \\ 0\end{array}\right]$. The maximum value of information delays are $\lambda_{i}^{\max }=1, i=1, \ldots, 6$. All agents are Schur stable and controllable.

The initial conditions are chosen equal to: $x_{1}(0)=[100 ; 100]^{\mathrm{T}}, x_{2}(0)=[60 ; 60]^{\mathrm{T}}, x_{3}(0)=[20 ; 20]^{\mathrm{T}}$, $x_{4}(0)=[-20 ;-20]^{\mathrm{T}}, x_{5}(0)=[-60 ;-60]^{\mathrm{T}}, x_{6}(0)=[-100 ;-100]^{\mathrm{T}}, x_{i}(-t)=x_{i}(0), i=1, \ldots, 6$, $t=1, \ldots, \lambda_{i}^{\max }$.

As a result of solving problem (18) under constraint (19), feedback control gain matrices are calculated for each of the agents in accordance with $(20)$ : $\mathrm{K}_{1}=[-0.547 ;-0.582], \mathrm{K}_{i}=[-0.702 ;-0.709]$, $i=2, \ldots, 6$. 
The vector (22) and general (23) Lyapunov functions were constructed for the considered system. The values of the elements of the dynamics matrix $\Lambda$ of the comparison system (24) are determined with accordance to [22]:

$$
\Lambda=\left[\begin{array}{cccccc}
0.731 & 0.111 & 0.169 & 0.111 & 0 & 0.127 \\
0.144 & 0.685 & 0.201 & 0 & 0 & 0 \\
0.062 & 0.056 & 0.631 & 0 & 0 & 0 \\
0.144 & 0 & 0 & 0.792 & 0.144 & 0 \\
0 & 0 & 0 & 0.111 & 0.646 & 0.127 \\
0.109 & 0 & 0 & 0 & 0.109 & 0.462
\end{array}\right]
$$

The eigenvalues of the dynamics matrix $\Lambda$ are $\{0.995 ; 0.831 ; 0.631 ; 0.595 ; 0.549 ; 0,345\}$, at that, condition (25) is fulfilled and matrix $\Lambda$ is Schur stable. Consequently, the networked MAS with given input delays is also stable.

The diagonal weight matrices of local criteria (5) are chosen $\mathrm{W}_{i}^{x}=\operatorname{diag}\{0.1 ; 0.1\}$ and $\mathrm{W}_{i}^{u}=0.1$, $i=1, \ldots, 6$; discount coefficient is $\beta=0.9$.

Example 1. The delay values $\lambda_{i j}(k) \in\{0,1\}, i, j=1, \ldots, 6, j \neq i$ in each period were randomly selected. The Fig. 2 shows graphs of the first state variable of each agent $x_{i 1}(k)$ and the residuals $v_{i 1}(k)=\sum_{j=1, j \neq i}^{N} d_{i j}\left(x_{i 1}(k)-x_{j 1}\left(k-\lambda_{i j}(k)\right)\right), i=1, \ldots, 6$ determined with accordance to the consensus protocol (2). Obviously, the decrease in values indicates the consensus attainment, that is, the control goal is achieved.
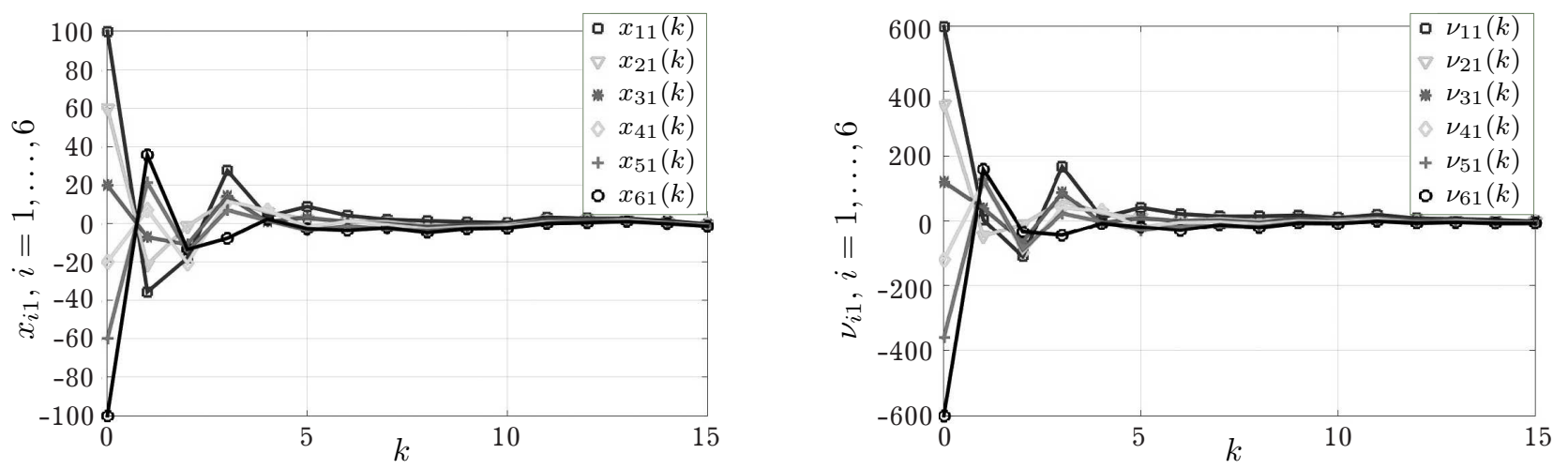

Fig. 2. Simulation results.

Example 2. A local set-up as a constant reference state $x_{1}^{*}=\operatorname{col}\{100 ; 0\}$ is applied to the state of the first agent. The Fig. 3 shows graphs of the first state variable of each agent and the residuals. Obviously, the consensus is also reached in this case.
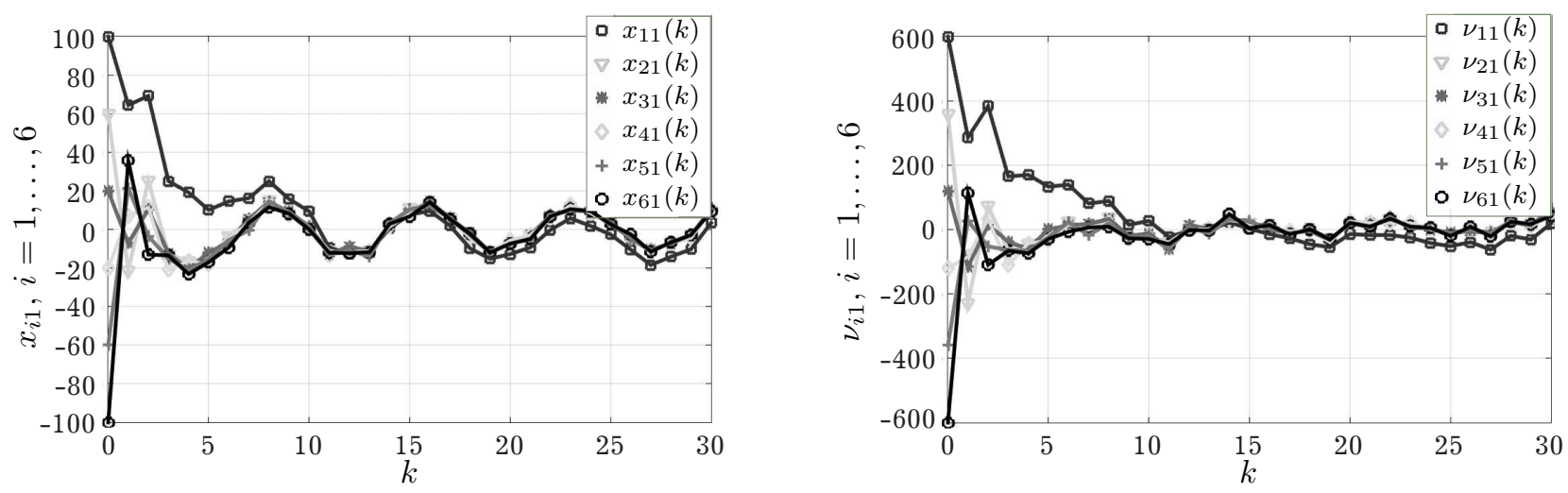

Fig. 3. Simulation results. 


\section{Conclusions}

In this paper, the consensus problem of the multi-agent systems with input delays is solved with the aid of the descriptor transformation of the closed-loop model and using the invariant ellipsoid method. The sufficient conditions are derived for the closed-loop descriptor model to achieve global consensus using Lyapunov-Krasovskii functional in the time domain. The obtained semidefinite programming problem is solved numerically using freely distributed software packages. The significance of this research is to provide a feasible method to deal with the consensus control for multi-agent systems with unknown but bounded input delays.

[1] Ren W., Beard R., Atkins E. A Survey of Consensus Problems in Multi-Agent Coordination. Proceedings of the 2005, American Control Conference. 3, 1859-1864 (2005).

[2] Olfati-Saber R., Fax A., Murray R. M. Consensus and Cooperation in Networked Multi-Agent Systems. Proceedings of the IEEE Int. Conference on Decision and Control. 95 (1), 215-233 (2007).

[3] Saber R. O., Murray R. M. Consensus Protocols for Networks of Dynamic Agents. Proceedings of the 2003 American Control Conference. 951-956 (2003).

[4] Mesbahi M., Egerstedt M. Graph theoretic methods in multiagent networks. New Jersey, Princeton University Press (2010).

[5] Olfati-Saber R., Murray R. M. Consensus Problems in Networks of Agents With Switching Topology and Time-Delays. IEEE Trans. Autom. Contr. 49 (9), 1520-1533 (2004).

[6] Bliman P. A., Ferrari-Trecate G. Average consensus problems in networks of agents with delayed communications. Automatica. 44 (8), 1985-1995 (2008).

[7] Munz U., Papachristodoulou A., Allgower F. Delay robustness in non-identical multi-agent systems. IEEE Transactions on Automatic Control. 57 (6), 1597-1603 (2012).

[8] Cao M., Morse A. S., Anderson B. D. O. Reaching a consensus in a dynamically changing environment: convergence rates, measurement delays, and asynchronous events. SIAM Journal on Control and Optimization. 47 (2), 601-623 (2008).

[9] Zuo Z., Wang C., Ding Z. A reduction method to consensus control of uncertain multi-agent systems with input delay. 2015 34th Chinese Control Conference (CCC). 7315-7321 (2015).

[10] Poznyak A., Polyakov A., Azhmyakov V. Attractive ellipsoids in robust control. Springer International Publishing (2014).

[11] Martynyuk A. A. Stability by Liapunov's Matrix Function Method with Applications. Marcel Dekker, Inc. (1998).

[12] Lakshmikantham V.V., Matrosov V. M., Sivasundaram S. Vector Lyapunov Functions and Stability Analysis of Nonlinear Systems. Netherlands, Springer (1991).

[13] Olfati-Saber R. Flocking for multi-agent dynamic systems: algorithms and theory. IEEE Transactions on Automatic Control. 51 (3), 401-420 (2006).

[14] Ren W., Beard R.W. Distributed Consensus in Multi-vehicle Cooperative Control. London, Springer (2008).

[15] Bauso D., Giarre L., Pesenti R. Non-linear protocols for optimal distributed consensus in networks of dynamic agents. Systems \& Control Letters. 55 (11), 918-928 (2006).

[16] Belov A. A., Kurdyukov A. P. Descriptor Systems and Control Problems. Moscow, Fizmatlit. (2015), (In Russian).

[17] Fridman E., Shaked U. A descriptor system approach to $\mathrm{H}_{\infty}$ control of linear time-delay systems. IEEE Transactions on Automatic Control. 47 (2), 253-270 (2002).

[18] Schneider R. Convex bodies: the Brunn-Minkowski theory. Cambridge University Press (1993).

[19] Polyak B. T., Hlebnikov M. V., Scherbakov P. S. Control of linear systems under external disturbances (linear matrix inequality technique. Moscow, LENAND (2014), (in Russian).

[20] Petersen I. R., McFarlane D. C., Rotea M. A. Optimal guaranteed cost control of discrete-time uncertain linear systems. Int. J. Robust Nonlinear Control. 8, 649-657 (1998).

Mathematical Modeling and Computing, Vol. 6, No. 2, pp. 333-343 (2019) 
[21] GrantM., BoydS. CVX: MATLAB software for disciplined convex programming, version 2.1. http://cvxr.com/cvx.

[22] Dorofieiev Y. I., LyubchykL. M., Nikulchenko A. A. Consensus decentralized control of multi-agent networked systems using vector Lyapunov functions. Proceedings of the 9th IEEE Intern. Conf. on Intelligent Data Acquisition and Advanced Computing Systems: Technology and Applications (IDAACS). 1, 60-65 (2017).

[23] Li Z., Duan Z. Cooperative control of multi-agent systems: a consensus region approach. Boca Raton, CRC Press (2017).

\title{
Консенсусне керування мультиагентними системами із запізнюваннями на вході: підхід на основі дескрипторної моделі
}

\author{
Любчик Л. М., Дорофєєв Ю. І. \\ Національний технічний університет "Харківсъкий політехнічний інститут", \\ вул. Кирпичова, 2, Харків, 61002, Україна
}

У статті розглянуто задачу консенсусного керування мультиагентними системами із запізнюваннями на вході, які є невідомими, але обмеженими. Дескрипторне перетворення використовується для отримання моделі замкнутого локального агента без запізнювань. За допомогою функціонала Ляпунова-Красовського визначено достатні умови досягнення глобального консенсусу в системі, де взаємодія між агентами відповідає заданій топології. Для аналізу стійкості мережі взаємозв'язаних агентів використано метод функцій Ляпунова, побудувавши відповідну систему порівняння. Ефективність запропонованого методу синтезу керування продемонстровано за допомогою чисельних прикладів.

Ключові слова: мультиагентна система, консенсусне керуваннл, дескрипторна модель, функиіонал Ляпунова-Красовсъкого, лінійна матрична нерівність, задача напіввизначеного програмування.

2000 MSC: 34K35, 90C22, 93D 30

Удк: 519.71 